\title{
LIVE VIRTUAL MACHINE MIGRATION USING SHADOW PAGING IN CLOUD COMPUTING
}

\author{
SangWook $\operatorname{Han}^{1}$ and HwaMin Lee ${ }^{2}$ \\ ${ }^{1}$ Department of Computer Science \&Engineering, Soonchunhyang University, \\ Asan, South Korea \\ sangukesch.ac.kr \\ ${ }^{2}$ Department of Computer Software Engineering, Soonchunhyang University, \\ Asan, South Korea \\ leehmesch.ac.kr
}

\begin{abstract}
Cloud Computing shares computing resources to execute application. Cloud systems provide high-specification resources in the form of services, leading to the provision of user convenience and greater ease for personal-computer users; however, expansions of the cloudsystem service necessitate a corresponding enhancement of the technology that is used for server-resource management. In this paper, by monitoring the resources of a cloud server, we sought to identify the causes of server overload and degradation, followed by the running of a dynamic-page-migration mechanism. According to this process, we designed the proposed migration architecture for the minimization of user inconvenience.
\end{abstract}

\section{KEYWORDS}

Live Migration, Shadow Paging, Dynamic Page Migration, Cloud Computing

\section{INTRODUCTION}

Cloud computing has garnered the spotlight over recent years in the field of computing. Cloud computing is tailored to the needs of the user, and its services are provided regardless of the location of the user and the devices that are used. The specifications that are required by a user or a corporation can be attained via WAN or Internet, in a home or office, in cafes, or on public transport; that is, you can use the service from any location [1].

Cloud computing can be divided into the following types: SaaS, PaaS, and IaaS; a computer user's choice will depend on the form that is needed. Regarding PaaS and SaaS, a computing environment must be provided for the user because the provision of hardware is too expensive in these cases; therefore, server visualization is used to reduce the cost [4]. Visualization involves the installation of a virtual machine monitor as systemic software that manages the virtualized operating systems on a single hardware unit, thereby supporting the virtual machine that is provided to the user, and the software is in the desired form for the installation of an operating system [1]. In terms of cloud computing, virtualization can be considered an application of the

Natarajan Meghanathan et al. (Eds) : NETCOM, NCS, WiMoNe, GRAPH-HOC, SPM, CSEIT - 2016 pp. 187- 193, 2016. ㄷ CS \& IT-CSCP 2016

DOI : $10.5121 /$ csit.2016.61516 
operating system. From the perspective of administrators, virtualization means that it is possible to deliver a service through the provision of an infrastructure and platform.

Although cloud computing is advantageous, however, two significant problems have emerged in relation to the use of virtual operating systems. First, hardware utilization is rapidly increased due to the frequent occurrence of page faults, leading to thrashing; and second, when a hardware failure occurs in the virtual machine, all of the corresponding services are interrupted. Resource management has become increasingly important because of these problems, and hardware-related research studies are in progress to develop measures that minimize user inconvenience without interrupting services. The purpose of this paper is the improvement of the efficiency of existing migration methods.

This paper is organized as follows: chapter 2 looks at the existing content regarding migration; chapter 3 proposes a mechanism for an efficient migration process through the use of monitoring and the dynamic-paging-migration technique; and in chapter 4 , the conclusion provides a direction for future research initiatives.

\section{RELATED WORKS}

\subsection{Monitoring}

The shapes and sizes of the clouds of cloud computing are widely variable. Because cloud users are difficult to manage personally, automation is required, and this necessitates an interaction with the surroundings. Monitoring can be seen as one of the best techniques for the configuration of a cloud system; by utilizing a monitoring system, it is possible for a user to perform the following actions, among others:

- Auto VM provisioning

- Auto scaling

- Auto service provisioning

- High availability

- Deploy management

\subsection{Hypervisor}

A hypervisor is the tool base for virtualization. Regarding commercial clouds, there is a variety of hypervisors that is divided into full virtualization and para-virtualization, depending on the virtualization method [2].

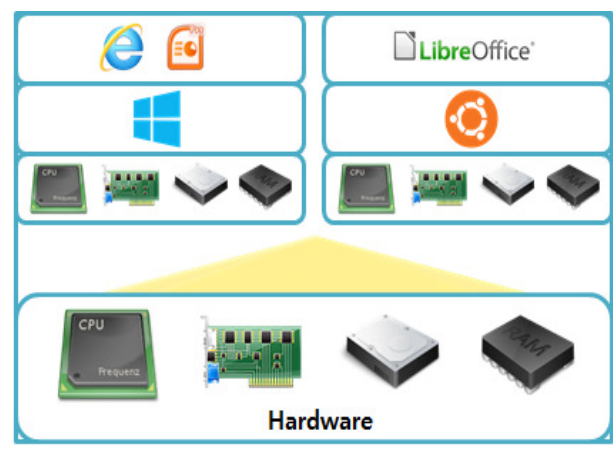

(a) Full virtualization

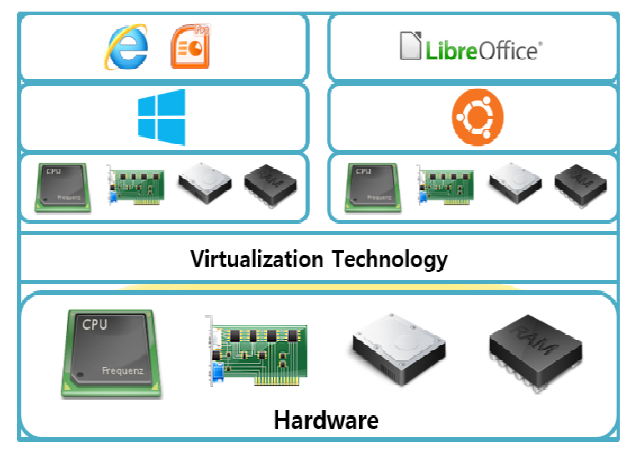

(b) Para virtualization

Figure 1. Types of Virtualization 
Full virtualization requires the virtualization of all of the hardware of a system, while the guest operating system remains unchanged, and the advantage is that it can be applied for a variety of operating systems.

As the name suggests, para-virtualization is applicable for a system wherein the hardware has not been fully virtualized; therefore, a guest operating system does not control the hardware.

\subsection{Shadow Paging}

Shadow Paging (Dynamic page migration) is a transition technique whereby a page is transmitted through the use of the physical memory space of a shadow page [1]. For the performance of a migration (the service runs on a virtual server), the information of the changed pages needs to be saved in the shadow page before the transferal to a newly allocated space can occur; therefore, this technique makes it possible to prevent service failure. Shadow paging involves the creation and saving of a physical memory space for the storage of the information of a page that has been changed by a user, and the newly created space is referred to as the "shadow page." Dynamic page migration comprises a mobile data system, whereby small amounts of memory space are progressively allocated to the target machine; by using the shadow page, mere seconds of service downtime occur, meaning that the user does not experience any inconvenience. The corresponding details are provided in chapter 3.2.

\subsection{Orchestration}

Orchestration is the management of resources whereby arrangement and alignment are automated. In terms of cloud-computing services, the following actions should be functional, among others: the issuance of an authentication key, the creation of a network check, and the creation of security rules. Orchestration cannot, however, be directly engaged each time an administrator creates an instance. A template-based engine can be used for the easy automation of this process that enables the deployment of infrastructure [2].

\section{VM LIVE MIGRATION TECHNIQUE}

\subsection{Monitoring-system configuration}

The previous monitoring system (Openstack ceilometer) offers the resources that need to be deployed in a cloud and enables a user to monitor the statuses of the resources for a performance assessment; furthermore, the program offers visibility and insight through the monitoring of the resource state of the dispersed cloud system [2]. The previous monitoring system, however, should change the internal source code according to systemic needs; therefore, we propose a monitoring system in this paper that can address this limitation. As shown in Table 1, the essential element of the monitoring system was selected.

Using the proposed monitoring system, it is possible to obtain a value for dynamic page migration by monitoring the information shown in Table 1 . In addition, the system can be used for the determination of the necessary information such as the page that needs to be sent when the information shown in Table 1 is obtained through the migration mechanism. 
Table 1. Information required for monitoring

\begin{tabular}{|l|}
\hline Edit page frequently \\
\hline Network transfer rate \\
\hline Page size \\
\hline Total size of virtual-machine memory \\
\hline
\end{tabular}

\subsection{Shadow-paging migration method}

If a server overload or degradation is detected through monitoring, the migration process is commenced. The corresponding migration-operation process is shown in Figure 3 and Table 2.

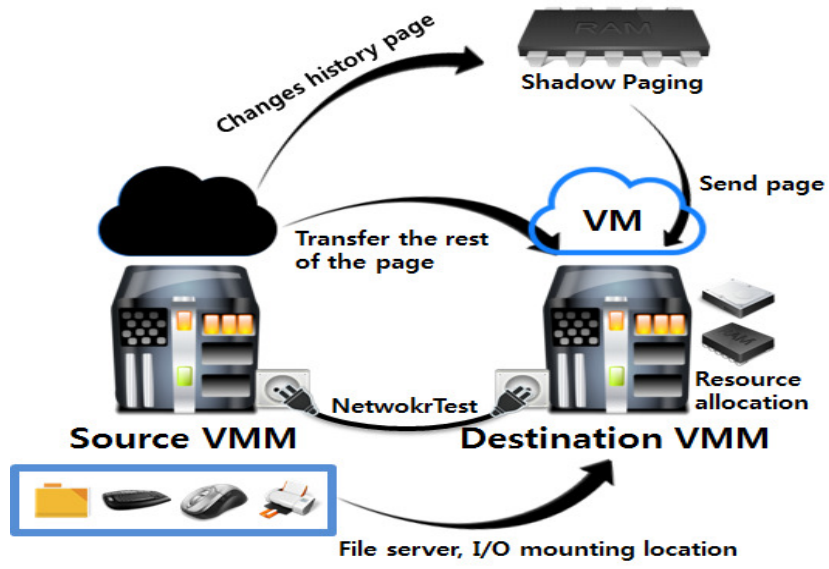

Figure 2. Proposed Shadow-paging Technique

A step-by-step description is shown in Table 2, as follows:

Table 2. Shadow-paging step-by-step instructions

1. Initialization phase :

- Target-machine selection

- Socket opening

2. Reservation phase :

- Connection of socket

- Allocation of a resource to the target machine

3. Iterative pre-copy phase :

- Storage of the changed information in the shadow page

4. Stop-and-copy phase:

- Sending of the rest of the page

5. Commitment phase :

- Mounting of the file server and I/O device to the target machine

6. Activation phase:

- Service activation 


\subsection{Full configuration for a virtualization migration}

In this paper, the flow of the proposed migration method is shown in Figure 4. If an overload or deterioration occurs in the server then the allocation of resources occurs quickly for the determination of the migration.

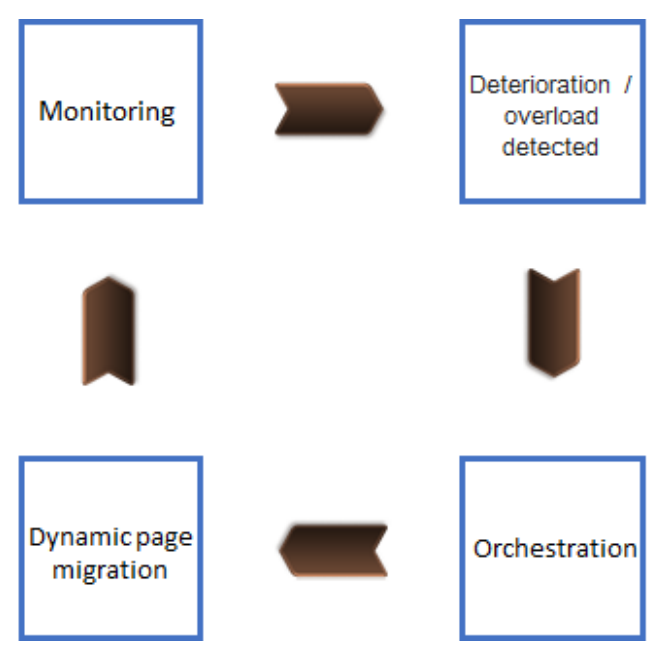

Figure 3. Proposed-migration-structure flow

The proposed monitoring system checks the status of the server, the Target-machine Selection Algorithm selects the target machine, and an automated orchestration process allocates the resources. By using the dynamic-page technique, the migration is performed.

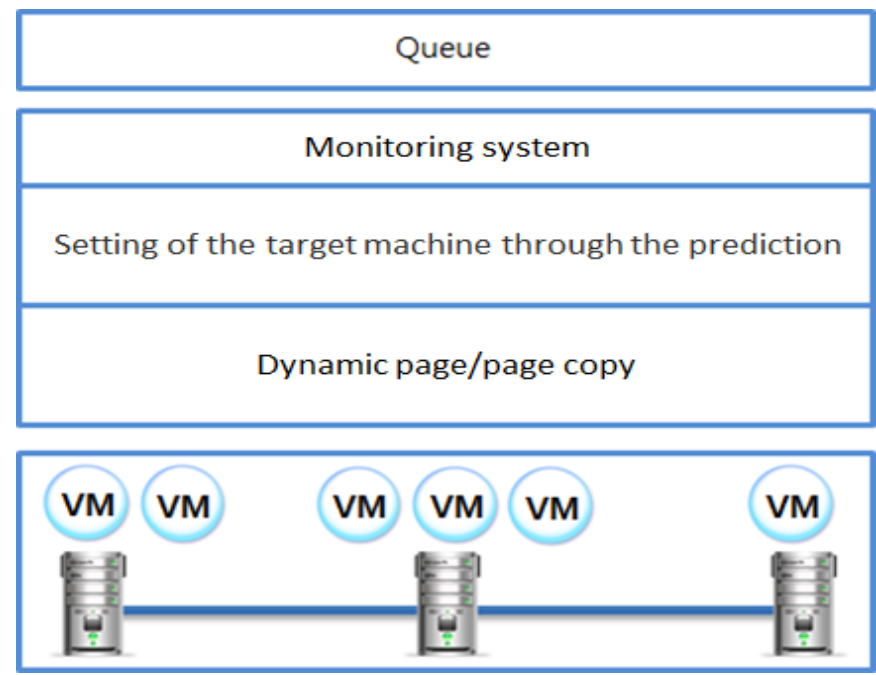

Figure 4. Proposed system structure

\section{CONCLUSION}

The development of cloud computing has led to many changes for both individuals and companies over recent years. Companies that provide cloud computing are therefore obliged to provide the best services to their customers and the companies in receipt of their services; furthermore, in addition to ensuring the effective management of their resources, they need to 
actively conduct research regarding migration. The method that is proposed in this research paper monitors resources to identify any deterioration or overloading regarding a server, and it performs migration in real time; provided that a target machine is nominated, this proposed method can be used for a server migration while the services are maintained by shadow paging. Further study is required regarding effective algorithms for shadow-paging migration; accordingly, this paper's objective is the development of a faster and more-precise migration technique for the harmonization of target-algorithm selection and the dynamic-page-migration algorithm.

\section{ACKNOWLEDGEMENTS}

This research was supported by the MSIP(Ministry of Science, ICT and Future Planning), Korea, under the ITRC(Information Technology Research Center) support program(IITP-2016-R099216-1006) supervised by the IITP(Institute for Information \& communications Technology Promotion).

\section{REFERENCES}

[1] Kapil, D., Pilli, E. S., \& Joshi, R. C. (2013) Live virtual machine migration techniques: Survey and research challenges, In 2013 IEEE 3rd International Advance Computing Conference, pp. 963-969.

[2] Son, A. Y., \& Huh, E. N. (2016) Migration Method for Seamless Service in Cloud Computing: Survey and Research Challenges, In 2016 30th International Conference on Advanced Information Networking and Applications Workshops (WAINA), pp. 404-409.

[3] Wood, T., Ramakrishnan, K. K., Shenoy, P., \& Van der Merwe, J. (2011) CloudNet: dynamic pooling of cloud resources by live WAN migration of virtual machines, In ACM Sigplan Notices, Vol. 46, No. 7, pp. 121-132.

[4] Kim, B., (2013) "An Efficient Method for Live Virtual Machine Migration Time Using Prediction of Pre-copy Phase", Master's Thesis, Seogang University, Korea.

[5] Du, Y., Yu, H., Shi, G., Chen, J., \& Zheng, W. (2010) Microwiper: efficient memory propagation in live migration of virtual machines. In 2010 39th International Conference on Parallel Processing, pp. 141-149.

[6] Ahmad, R. W., Gani, A., Hamid, S. H. A., Shiraz, M., Yousafzai, A., \& Xia, F. (2015). A survey on virtual machine migration and server consolidation frameworks for cloud data centers. Journal of Network and Computer Applications, 52, 11-25.

[7] Zheng, J., Ng, T., Sripanidkulchai, K., \& Liu, Z. (2013). Pacer: A progress management system for live virtual machine migration in cloud computing.IEEE transactions on network and service management, 10(4), 369-382.

[8] Nathan, S., Bellur, U., \& Kulkarni, P. (2015, August). Towards a comprehensive performance model of virtual machine live migration. In Proceedings of the Sixth ACM Symposium on Cloud Computing (pp. 288-301). ACM.

[9] Sun, G., Liao, D., Anand, V., Zhao, D., \& Yu, H. (2016). A new technique for efficient live migration of multiple virtual machines. Future Generation Computer Systems, 55, 74-86.

[10] Jackson, K., Bunch, C., \& Sigler, E. (2015). OpenStack cloud computing cookbook. Packt Publishing Ltd. 


\section{AUTHORS}

\section{Sangwook Han}

He is master course student at Dept. of Computer Software Engineering in Soonchunhyang University. His main research interests are cloud and mobile computing.

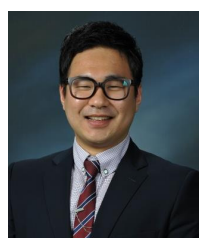

\section{HwaMin Lee}

She is a Professor at Dept. of Computer Software Engineering in Soonchunhyang University. Her main research interests are cloud and mobile computing, Internet of Things, and IT convergence.

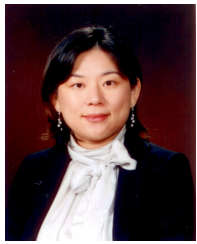

